UCRL-CR--104962

DE91 000951

$(11,1) 1090$

NOTE ON SUFFICIENT SYMMETRY CONDITIONS

FOR ISOTROPY OF THE ELASTIC MODULI TENSOR

\author{
M.S. Dresselhaus \\ G. Dresselhaus
}

August 29, 1990
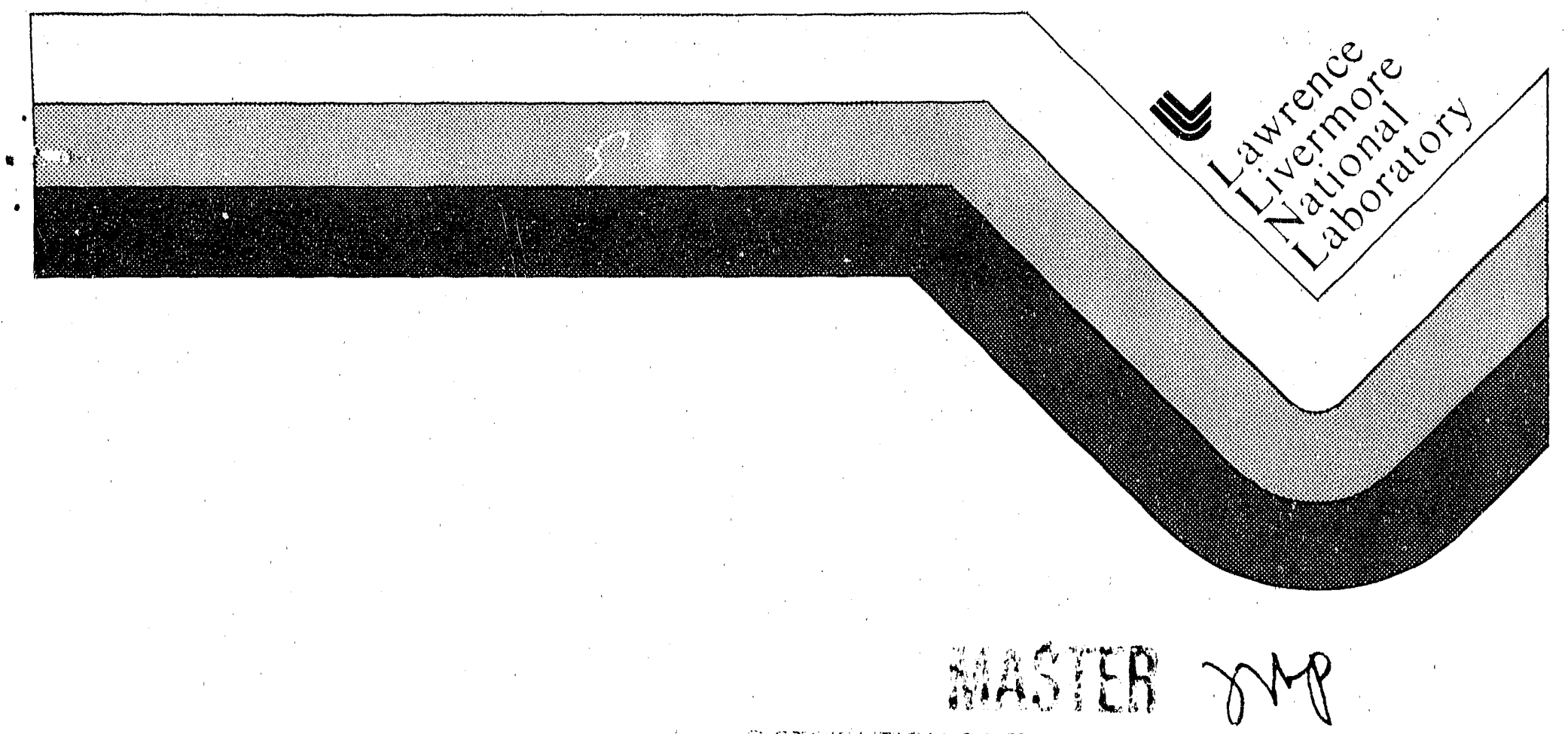

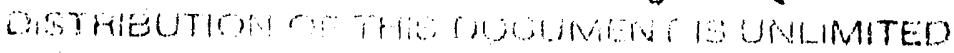


DISCLAIMER

Work performed under the auspices of the U.S. Departme'nt of Energy by I awrence Livermore National Laboratory under contract number W-7405-ENG-48.

This dorument was prepared as an account of work sponsored by an agency of the United States Government. Neither the United States Government nor the University of California nor any of their employees, makes any warranty. express or implied, or assumes any legal liability or responsibility for the accuracy, completeness, or usefulness of any information, apparatus, product, or process disclosed, or represents that its use would not infringe privately owned rights. Reference herein to any specific commercial products, process, or service by trade name, Irademark, manufacturer, or otherwise, does not necessarily constitute or imply its endorsement, recommendation, or favoring by the United States Government or the University of California. The views and opinions of authors expressed herein du not necessarily state or reflect those of the United States Government or the University of California, and shall not be used for advertising or product endorsement purposes. 


\title{
Note on Sufficient Symmetry Conditions for Isotropy of the Elastic Moduli Tensor
}

\author{
M. S. Dresselhaus ${ }^{\dagger}$ \\ Department of Electrical Engineering and Computer Science and Department of Physics, \\ Massachusetts Institute of Technology, Combridge, Massachusetts 02139 \\ and \\ G. Dresselliaus \\ Francis Bitter National Magnet Laboratory, \\ Massachusetts Institute of Technology, Cambridge, Massachusetts 02139
}

(Received August 29, 1990)

Group theoretical methods are used to obtain the form of the elastic moduli matrices and the number of independent parameters for various symmetries. Particular attention is given to symmetry groups for which 3D and 2D isotropy is found for the stress-strain tensor relation. The number of independent parameters is given by the number of times the fully symmetric representation is contained in the direct product of the irreducible representations for two symmetrical second rank tensors. The basis functions for the lower symmetry groups are found from the compatibility relations and are explicitly related to the elastic moduli. These types of symmetry arguments should be generally useful in treating the elastic properties of solids and composites.

62.20.Dc, 81.40.Jj,03.40.Dz 


\section{INTRODUCTION}

Christensen has shown. ${ }^{1}$ that the 4 th rank symmetric elastic tensor $\mathcal{C}_{i j k l}$ defined by

$$
\frac{\partial F_{i}}{\partial r_{j}}=\sum_{k, l=x, y, z} \mathcal{C}_{i j k l} e_{k l}
$$

where $F_{i}$ is the component of the force in direction $r_{i}$, retains its form in $3 \mathrm{D}$ in going from the isotropic full rotational symmetry group to the case of 6 five-fold rotation axes. Christensen further obtained the corresponding result in $2 \mathrm{D}$ where the form of the $\mathcal{C}_{i, j l}$ is retained in going from the case of full axial symmetry (in-plane isotropy) to hexagonal symmetry with a 6 -fold axis. In his paper Christensen ${ }^{1}$ emphasizes that the more restricted symmetry of the 6 five-fold axes in the $3 D$ case, and the hexagonal axis in the $2 \mathrm{D}$ case is sufficient to yield $3 \mathrm{D}$ and $2 \mathrm{D}$ isotropy with regard to the elastic properties, defined by Eq. 1.

Fiber reinforced composites represent an interesting application of these symmetry forms. ${ }^{1}$ If the fibers are oriented in three dimensional space in the six directions prescribed by icosahedral symmetry, then isotropy of the tensor of elastic moduli will be obtained. This possibility was first suggested by Rosen. ${ }^{2}$ In the corresponding two dimensional situation, if the fibers are oriented at $60^{\circ}$ intervals then isotropy is obtained in the plane. It is standard practice to use fiber composite sheets stacked at $60^{\circ}$ angular intervals to obtain "quasi-isotropy" in the field of fiber composites. Recent, research on quasi-crystals ${ }^{3,4}$ has emphasized the connection of the icosahedral symmetry to the elastic properties. 3,4

In this note we show a generalization of Christensen's proof, using group theoretical arguments. From the group theoretical point of view presented here, the conditions for retaining isotropy can be clearly defined and when the symmetry is further lowered to introduce anisotropy in the elastic properties, the passage from 
the isotropic to the anisotropic situations can be followed adiabatically through use

of compatibility relations. For those with a group theory background, the results of this note are almost obvious, but for materials scientists without this background these results do not seem so obvious. The objective of this note then is to make certain symmetry results available to materials scientists for use in their applications.

\section{3D ISOTROPY}

For the case of full rotational symmetry, a second rank tensor $\stackrel{\leftrightarrow}{T}$ transforms according to the representation $\Gamma_{\vec{T}}$ where $\Gamma_{\vec{T}}$ can be written as a symmetric and an antisynmetric part

$$
\Gamma_{\overparen{T}}=\Gamma_{\vec{T}}^{(s)}+\Gamma_{\stackrel{T}{(a)}}^{(a)}
$$

where the symmetric components transform as the irreducible representations

$$
\Gamma_{T}^{(s)}=\Gamma_{l=0}+\Gamma_{l=2}
$$

and the antisymmetri components transform as

$$
\Gamma_{\vec{T}}^{(a)}=\Gamma_{l=1}
$$

in which the irreducible representations of the full rotation group are denoted by their total angular momentum values $l$. Since the stress $\vec{\nabla} \cdot \vec{F}$ and strain $\stackrel{\leftrightarrow}{e}$ tensors are symmetric second rank tensors, both $X_{\alpha \alpha}$ and $e_{i j}$ transform according to $\left(\Gamma_{l=0}+\Gamma_{l=2}\right)$ in full rotational symmetry, where $X_{\alpha}$ denotes a force in the $x$ direction applied to a plane whose normal is in the $\alpha$ direction. The fourth rank symmetric $\mathcal{C}_{i j k l}$ tensor of $\mathrm{E}_{1} .1$ transforms according to the symmetric part of the direct product of two second rank symmetric tensors $\Gamma_{\vec{e}}^{(s)} \otimes \Gamma_{\vec{e}}^{(s)}$ yielding

$$
\left(\Gamma_{l=0}+\Gamma_{l=2}\right) \otimes\left(\Gamma_{l=0}+\Gamma_{l=2}\right)=\left(2 \Gamma_{l=0}+2 \Gamma_{l=2}+\Gamma_{l=4}\right)^{(s)}+\left(\Gamma_{l=1}+\Gamma_{l=2}+\Gamma_{l=3}\right)^{(a)},
$$


so that in general $e_{i j}$ is specified by 6 constants and the $\mathcal{C}_{i j k l}$ tensor by 21 constants because it is symmetrical under the interchange of ij $\rightarrow k l$. The additional 15 constants that specify the antisymmetric off-diagonal irreducible representations are not needed to specify the $\mathcal{C}_{i j k l}$. In the case of full rotational symmetry, Eq. 5 shows that the totally symmetric representation $\left(\Gamma_{l=0}\right)$ is contained only twice in the direct product of the irreducible representations for two second rank symmetric tensors, indicating that only two independent non-vanishing constants are needed to describe the 21 constants of the $\mathcal{C}_{i j k l}$ tensor, a resuit that is well known in elasticity theory for isotropic media. In general, the number of times the totally symmetric representation (e.g., $\Gamma_{l=0}$ for the full rotational group) is contained in the irreducibìe representations of a general matrix of arbitrary rank gives the number of independent non-vanishing constants needed to specify that matrix.

We denote the two independent non-vanishing constants needed to specify the $\mathcal{C}_{i j k l}$ tensor by $C_{0}$ for $\Gamma_{l=0}$ and by $C_{2}$ for $\Gamma_{l=2}$ symmetry. We then use these two constants to relate symmetrized stresses and strains labeled by the irreducible representations $\Gamma_{l=0}$ and $\Gamma_{l=2}$ in the full rotation group. The symmetrized stress-strain equations are first written in full rotational symmetry, using the partners of the irreducible representations (one for $l=0$ and five for the $l=2$ partners):

$$
\begin{array}{cll}
\left(X_{x}+Y_{y}+Z_{z}\right)=C_{0}\left(e_{x x}+e_{y y}+e_{z z}\right) & \text { for } & l=0, m=0 \\
\left(X_{x}-Y_{y}+i Y_{x}+i X_{y}\right)=C_{2}\left(e_{x x}-e_{y y}+i e_{x y}+i e_{y x}\right) & \text { for } & l=2, m=2 \\
\left(Z_{x}+X_{z}+i Y_{z}+i Z_{y}\right)=C_{2}\left(e_{z x}+e_{z x}+i e_{y z}+i e_{z y}\right) & \text { for } & l=2, m=1 \\
\left(Z_{z}-\frac{1}{2}\left(X_{x}+Y_{y}\right)\right)=C_{2}\left(e_{z z}-\frac{1}{2}\left(e_{x x}+e_{y y}\right)\right) & \text { for } & l=2, m=0 \\
\left(Z_{x}+X_{z}-i Y_{z}-i Z_{y}\right)=C_{2}\left(e_{z x}+e_{x z}-i e_{y z}-i e_{z y}\right) & \text { for } & l=2, m=-1 \\
\left(X_{x}-Y_{y}-i Y_{x}-i X_{y}\right) & =C_{2}\left(e_{x x}-e_{y y}-i e_{x y}-i e_{y x}\right) \text { for } & l=2, m=-2
\end{array}
$$

From $\mathrm{Eq}_{1} .6$ we solve for the six independent stress coefficients in terms of the strains, yielding 


$$
X_{x}=\left(\frac{C_{0}}{3}+\frac{2 C_{2}}{3}\right) e_{x x}+\left(\frac{C_{0}}{3}-\frac{C_{2}}{3}\right)\left(c_{y y}+e_{z z}\right)
$$

for the stress component $X_{x}$. Five additional relations are then written down for the other 5 itress components.

In the notation that is commonly used, we write the stress-strain relations

$$
\sigma_{i}=\sum_{j=1,6} C_{i j} \varepsilon_{j}
$$

where the 6 components of the symmetric stress and strain tensors are written as

$$
\begin{array}{lll}
\sigma_{1}=X_{x} & \varepsilon_{1}=e_{x x} \\
\sigma_{2}=Y_{y} & \varepsilon_{2}=e_{y y} \\
\sigma_{3}=Z_{z} & \varepsilon_{3}=e_{z z} \\
\sigma_{4}=\frac{1}{2}\left(Y_{z}+Z_{y}\right) & \text { and } \quad & \varepsilon_{4}=\left(e_{y z}+e_{z y}\right) \\
\sigma_{5}=\frac{1}{2}\left(Z_{x}+X_{z}\right) & \varepsilon_{5}=\left(e_{z x}+e_{x z}\right) \\
\sigma_{6}=\frac{1}{2}\left(X_{y}+Y_{x}\right) & \varepsilon_{6}=\left(e_{x y}+e_{y x}\right)
\end{array}
$$

and $C_{i j}$ is the $6 \times 6$ elastic moduli matrix $C_{i j}$. In this notation the 21 partners that transform as $\left(2 \Gamma_{l=0}+2 \Gamma_{l=2}+\Gamma_{l=4}\right)$ in $E_{1} .5$ correspond to the symmetric partners while the 15 partners in $\left(\Gamma_{l=1}+\Gamma_{l=2}+\Gamma_{l=3}\right)$ correspond to the antisymmetric partners that do not give rise to any independent elastic components, since $C_{i j}=C_{j i}$. From the six relations for the six stress components (one of which is given explicitly by Eq. 7), the relations between the $C_{0}$ and $C_{2}$ and the $C_{i j}$ follow:

$$
\begin{aligned}
& C_{11}=\frac{1}{3}\left(C_{0}+2 C_{2}\right)=C_{22}=C_{33} \\
& C_{12}=\frac{1}{3}\left(C_{0}-C_{2}\right)=C_{13}=C_{23}^{\prime} \\
& C_{44}=\frac{1}{2} C_{2}=C_{55}=C_{66}
\end{aligned}
$$

from which we construct the $C_{i, j}$ matrix for a $3 \mathrm{D}$ isotropic medium involving two independent constants $C_{11}$ and $C_{12}$ 


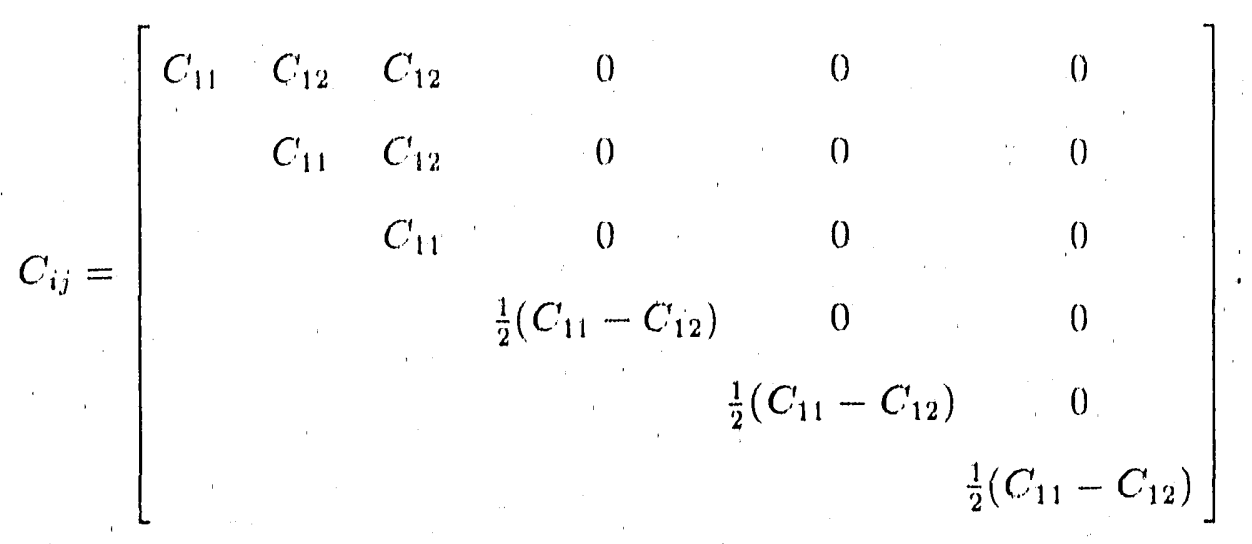

Any subgroup of the full rotation group for which the 5-fold $\Gamma_{l=2}$ level degeneracy is not lifted will leave the form of the $C_{i j}$ matrix mvariant, thus giving a more general proof of Christensen's arguments. ${ }^{1}$ The icosuhedral group with inversion symmetry $I_{h}$, which is a subgroup of the full rotation group, and the icosahedral group without inversion $I$, which is a subgroup of both the full rotation group and the group $I_{h}$, are two examples of groups which preserve the 5-told degenerate level and hence retain the form of the $C_{i j}$ matrix given by $E_{q}$. 11 . This result follows from at least two related arguments. Firstly, from the pertinent compatibility relations between the full rotation group ${ }^{5,6}$ and the $I_{h}$ group (see Table I for the character table), $f$

$$
\begin{aligned}
& \Gamma_{l=0} \longrightarrow\left(A_{g}\right)_{I_{h}} \\
& \Gamma_{l=2} \longrightarrow\left(H_{g}\right)_{I_{h}}
\end{aligned}
$$

we show that

$$
\Gamma_{\vec{c}}^{(s)}=\left(A_{g}\right)_{l_{h}}+\left(H_{g}\right)_{I_{h}} .
$$

From Eq. 13 we see that no lifting of degeneracy occurs in going from full rotational symmetry to $I_{h}$ symmetry from which it follows that the number of non-vanishing independent constants in the $C_{i j}$ matrix remains at 2 for $I_{h}$ (and $I$ ) symmetry.

The sane conclusion follows from the fact that the basis functions for $\Gamma_{l=0}$ and $\Gamma_{l=2}$ for the full rotation group can also be used as basis functions for the $A_{g}$ and 
$H_{y}$ irreducible representations of $I_{h}$. Therefore the same stress-strain rolations are obtained in $I_{h}$ symmetry as are given in $\mathrm{Eq}_{\mathrm{g}}$. 6. It therefore follows that the form of the $C_{i j}$ matrix will also be the same for $I_{h}$ and full rotational symmetry, thereby completing the proof.

Clearly, the direct product $\Gamma_{\vec{e}}^{(s)} \otimes \Gamma_{\vec{e}}^{(s)}$ given by Eq. 5 is not invariant as the symmetry is reduced from full rotational symmetry to $I_{h}$ symmetry since the 9-fold representation $\Gamma_{l=4}$ in Eq. 5 splits into the irreducible representations $\left(G_{g}+H_{g}\right)$ in going to the lower symetry group $I_{h}$. But this is not of importance to the linear stress-strain equations which are invariant to this lowering of symmetry. It might be worth mentioning here that when non-linear effects are taken into account and perturbations from Eq. 1 are needed to specify the stress--strain relations (for example, tems in the strain squared), different mechanical behavior would be expected to occur in $I_{h}$ symmetry in comparison with the full rotation group. In such a case, the compatibility relations between the full rotation group and the $I_{h}$ group can be used to relate the terms in the generalized elastic moduli matrix for the two symmetrics.

It should be noted that all symmetry groups forming Bravais lattices in solid state physics have too few symmetry operations to preserve the 5-fold degeneracy of the $l=2$ levd; for example in cubic $O_{h}$ symmetry the cubic group with the highest symmetry, the $l=2$ level corresponds to a reducible representation of group $O_{h}$ which splits into a 3 -fold and a 2 -fold level (the $T_{2 y}$ and $E_{y}$ levels), so that in this case (see Eq. 6), 3 elastic constants are needed to specify the $6 \times 6$ matnix for $C_{i j}$ 


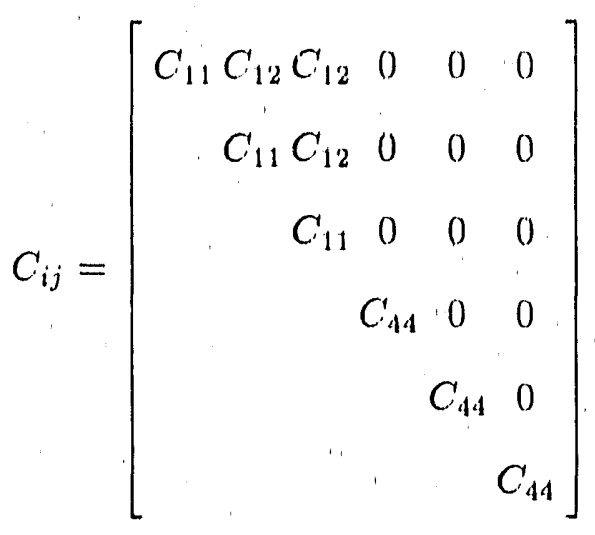

as is described in many solid state physics books, 7,8

\section{2D ISOTROPY}

A similar situation applies in 2D, Here the full axial symmetry is described by the group $D_{\infty}$. The irreducible representations of $D_{\infty}$ that ase contained in the symmetric second rank tensor are

$$
\Gamma_{\vec{e}}^{(s)}=2 A_{1 g}+E_{1 g}+E_{2 g}
$$

so that the symmetric part of the direct product becomes

$$
\left(\Gamma_{\vec{e}}^{(s)} \otimes \Gamma_{\vec{e}}^{(s)}\right)^{(s)}=5 A_{1 g}+3 E_{1 g}+3 E_{2 g}+E_{3 g}+E_{4 y},
$$

indicating that the $C_{i j}$ matrix can be described in terms of 5 independent constants for full axial symmetry. The stress-strain relations for $D_{\text {coh }}$ symmetry are written in symmetrized form using the basis functions

$$
\begin{array}{cc}
\left(X_{x}+Y_{y}+Z_{z}\right) \text { and }\left(e_{x x}+e_{y y}+e_{z z}\right) & \text { for } l=0, m=0 \quad A_{1 y} \\
\left(X_{x}-Y_{y}+i Y_{x}+i X_{y}\right) \text { and }\left(e_{x x}-e_{y y}+i e_{x y}+i e_{y x}\right) \text { for } l=2, m=2 \quad E_{2 y} \\
\left(X_{x}-Y_{y}-i Y_{x}-i X_{y}\right) \text { and }\left(e_{x x}-e_{y y}-i e_{x y}-i e_{y x}\right) \text { for } l=2, m=-2 & E_{2 y} \\
\left(Z_{x}+X_{z}+i Y_{z}+i Z_{y}\right) \text { and }\left(e_{z x}+e_{x z}+i e_{y z}+i e_{z y}\right) & \text { for } l=2, m=1 \quad E_{1 y} \\
\left(Z_{x}+X_{z}-i Y_{z}-i Z_{y y}\right) \text { and }\left(e_{z x}+e_{x z}-i e_{y z}-i e_{z y}\right) \text { for } l=2, m=-1 & E_{1 y} \\
\left(Z_{z}-\frac{1}{2}\left(X_{x}+Y_{y}\right)\right) \text { and }\left(e_{z z}-\frac{1}{2}\left(e_{x x}+e_{y y}\right)\right) & \text { for } l=2, m=0 \quad A_{l y}
\end{array}
$$


yiolding,

$$
\begin{aligned}
X_{x}+Y_{y}+Z_{z} & =C_{A_{1,}, 1}\left(e_{x x}+e_{y y}+e_{z z}\right)+C_{A_{1, y},}\left[e_{z z}-\frac{1}{2}\left(e_{y x}+e_{y y}\right)\right] \\
Z_{z}-\frac{1}{2}\left(X_{x}+Y_{y}\right) & =C_{A_{1,}, 2}\left[e_{z z}-\frac{1}{2}\left(e_{x w}+e_{y y}\right)\right]+C_{A_{1,}, 4}\left[e_{x w}+e_{y y}+e_{z z}\right] \\
X_{x}-Y_{y} & =C_{E_{2 y}}\left(e_{x w}+e_{y y}\right)
\end{aligned}
$$

and corresponding equations for $X_{y}, Y_{z}$ and $X_{z}$. We then solve Eq. 18 for $X_{x}, Y_{y}$ and $Z_{z}$ and require $C_{i j}=C_{j i}$. In the case of $D_{\infty i h}$, the requirement that $C_{31}=$ $C_{13}=C_{32}=C_{23}$ yields the additional constraint $C_{A_{1 g}, 3}=2 C_{A_{1 g}, 4}$ which is needed to obtain the 5 independent symmetry coefficients as required by $\mathrm{Eq}$. 16: $C_{A_{1 g}, 1}$, $C_{A_{1 y}, 2}, C_{A_{1 g}, 3}, C_{E_{1,}}$ and $C_{E_{2 g}}$. The relations between these symmetry coefficients and the $C_{i j}$ coefficients are:

$$
\begin{aligned}
& C_{11}=C_{22}=\frac{1}{2}\left[\frac{2}{3} C_{A_{19}, 1}+\frac{1}{3} C_{A_{1 g}, 2}-\frac{2}{3} C_{A_{1 g}, 3}+C_{E_{2 g}}\right] \\
& C_{12}=C_{21}=\frac{1}{2}\left[\frac{2}{3} C_{A_{1 g}, 1}+\frac{1}{3} C_{A_{1 g}, 2}-\frac{2}{3} C_{A_{1 g}, 3}-C_{E_{2 g}}\right] \\
& C_{13}=C_{23}=\frac{1}{3}\left[C_{A_{1 g}, 1}-C_{A_{1 g}, 2}+\frac{1}{2} C_{A_{1 g}, 3}\right] \\
& C_{33}=\frac{1}{3}\left[C_{A_{1 g}, 1}+2 C_{A_{1,}, 2}+2 C_{A_{1 g}, 3}\right] \\
& C_{44}^{\prime}=C_{55}=\frac{1}{2} C_{E_{1 g}} \\
& C_{6 S}^{\prime}=\frac{1}{2} C_{E_{29}}=\frac{1}{2}\left(C_{22}-C_{21}\right)=\frac{1}{2}\left(C_{11}-C_{12}\right) .
\end{aligned}
$$

Combining the non-vanishing $C_{i j}$ coefficients then yields the matrix for full axial symmetry $D_{\infty / 2}$

$$
C_{i j}=\left[\begin{array}{cccccc}
C_{11} & C_{12} & C_{13} & 0 & 0 & 0 \\
& C_{11} & C_{13} & 0 & 0 & 0 \\
& & C_{33} & 0 & 0 & 0 \\
& & & C_{44} & 0 & 0 \\
& & & & C_{44} & 0 \\
& & & & & \frac{1}{2}\left(C_{11}-C_{12}\right)
\end{array}\right]
$$


Once again, the basis functions used to obtain the stress-strain relations also serve as basis functions for the irreducible representations $A_{1 g}, E_{1 g}$, and $E_{2 g}$ in the hexagonal group $D_{6 h}$ which is a subgroup of $D_{\infty h}$. Thus the stress-strain relations for $D_{6 h}$ symmetry are identical to $D_{\infty h}$ and the same form of the $6 \times 6$ elastic constant matrix $C_{i j}$ follows, completing a generalization of the result proven by Christensen in $2 \mathrm{D} .{ }^{1}$

If we consider the subgroup of $D_{\infty h}$ with the next highest symmetry $\left(D_{4 h}\right)$, we immediately see that there is only one 2-dimensional irreducible representation ${ }^{5,6}$ in $D_{4 h}$ so that the irreducible representations contained in second rank tensor $\Gamma_{\vec{e}}$ are not invariant as the symmetry is reduced from $D_{\infty h}$ to $D_{4 h}$.

It must be emphasized that in going from full rotational symmetry to $I_{h}$ symmetry or in going from $D_{\infty h}$ to $D_{6 h}$, the number of symmetry operations goes from $\infty$ to a relatively small number (120 for $I_{h}$ and 24 for $D_{6 h}$ ), so that some relations involving tensors of rank higher than 2 (such as the electrooptic coefficients) are no longer invariant under this lowering of symmetry both in $3 \mathrm{D}$ and in 2D.

\section{ACKNOWLEDGMENT}

We thank Dr. Richard Christensen of the Lawrence Livermore Laboratory for pointing out this problem and for numerous stimulating conversations. We also thank Prof. B. Budiansky of Harvard University for discussions of his unpublished proof, which uses similar arguments but is not expressed in group theory formalism. We gratefully acknowledge Subcontract No: B-130530 from Lawrence Livermore Laboratory for support of this research. 


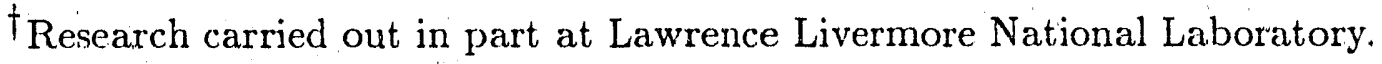

$\ddagger_{\text {Note that since Ref. }}{ }^{5,6}$ and other standard references do not list the character tables for the icosahedral group $I$ and $I_{h}$, we have included a character table for the group $I_{h}$.

${ }^{1}$ R.M. Christensen, J. Appl. Mechanics 54, 772 (1987).

${ }^{2}$ B.W. Rosen and L.S. Shu, J. Composite Mater. 5, 279 (1971).

${ }^{3}$ D. Levine, T.C. Lubensky, S. Ostlund, S. Ramaswamy, P.J. Steinhardt and J. Toner, Phys. Rev. Lett. 54, 1520 (1985).

${ }^{4}$ P.J. Steinhardt and S. Ostlund, "Physics of Quasi-Crystals", World Scientific Publishing, Singapore, Chap. 6 (1987).

${ }^{5}$ G.F. Koster, J. Dimmock and H. Statz, "Properties of the 32 point groups", MIT press, Cambridge, MA (1963).

${ }^{6}$ M. Tinkham, "Group Theory", McGraw Hill, NY (1963).

${ }^{` C}$. Kittel, "Introduction to Solid State Physics", John Wiley and Sons, NY, (1053).

${ }^{8}$ J.F. Nye, "Physical properties of crystals", Oxford, Clarendon Press (1957). 
TABLE I. Character table for $I_{h}$.

\begin{tabular}{|c|c|c|c|c|c|c|c|c|c|c|c|}
\hline$I_{h}$ & $E$ & $12 C_{5}$ & $12 C_{5}^{2}$ & $20 C_{3}$ & $15 C_{2}$ & $i$ & $12 S_{10}$ & $12 S_{10}^{3}$ & $20 S_{10}$ & $15 \sigma$ & Basis functions \\
\hline$A_{g}$ & 1 & 1 & 1 & 1 & 1 & 1 & 1 & 1 & 1 & 1 & $x^{2} y^{2} z^{2}$ \\
\hline$F_{1 g}$ & 3 & $\tau$ & $1-\tau$ & 0 & -1 & 3 & $1-\tau$ & $\tau$ & 0 & -1 & $R_{x}, R_{y}, R_{z}$ \\
\hline$F_{2 g}$ & 3 & $1-\tau$ & $\tau$ & 0 & -1 & 3 & $T$ & $1-\tau$ & 0 & -1 & \\
\hline$G_{g}$ & 4 & -1 & -1 & 1 & 0 & 4 & -1 & -1 & 1 & 0 & $\left\{\begin{array}{c}2 z^{2}-x^{2}-y^{2} \\
x^{2}-y^{2}\end{array}\right.$ \\
\hline$H_{g}$ & 5 & 0 & 0 & -.1 & 1 & 5 & 0 & 0 & -1 & 1 & $\left\{\begin{array}{l}x y \\
x z \\
y z\end{array}\right.$ \\
\hline$A_{u}$ & 1 & 1 & 1 & 1 & 1 & -1 & -1 & -1 & -1 & -1 & \\
\hline$F_{1 u}$ & 3 & $\tau$ & $1-\tau$ & 0 & -1 & -3 & $\tau-1$ & $-\tau$ & 0 & 1 & $(x, y, z)$ \\
\hline$F_{2 u}$ & 3 & $1-\tau$ & $\tau$ & 0 & -1 & -3 & $-\tau$ & $\tau-1$ & 0 & 1 & $\begin{array}{l}\left(x^{3}, y^{3}, z^{3}\right) \\
\left(x\left(z^{2}-y^{2}\right)\right.\end{array}$ \\
\hline$G_{u}$ & 4 & -1 & -1 & 1 & 0 & -4 & 1 & 1 & -1 & 0 & $\left\{\begin{array}{c}y\left(z^{2}-x^{2}\right) \\
z\left(x^{2}-y^{2}\right) \\
x y z\end{array}\right.$ \\
\hline$H_{u}$ & 5 & 0 & 0 & -1 & 1 & -5 & 0 & 0 & 1 & -1 & \\
\hline
\end{tabular}

where $\tau=(1+\sqrt{5}) / 2$ and is often referred to as the "golden mean". 

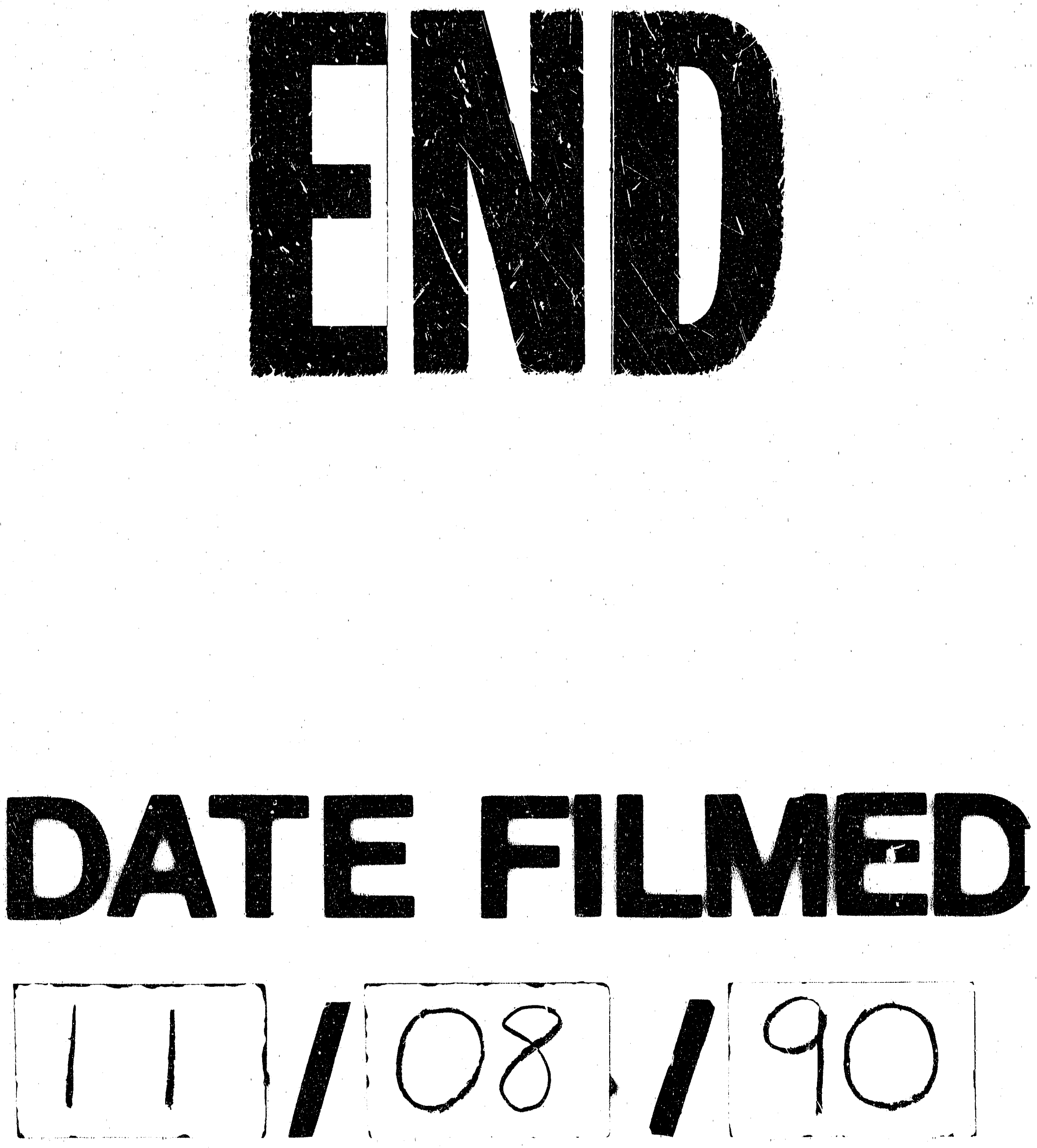
